\title{
Solving Exist-Random Quantified Stochastic Boolean Satisfiability via Clause Selection
}

\author{
Nian-Ze Lee ${ }^{* 1}$, Yen-Shi Wang ${ }^{* 2}$ and Jie-Hong R. Jiang ${ }^{1,2}$ \\ ${ }^{1}$ Graduate Institute of Electronics Engineering, National Taiwan University, Taipei, Taiwan \\ ${ }^{2}$ Department of Electrical Engineering, National Taiwan University, Taipei, Taiwan \\ \{d04943019, b03901116, jhjiang\}@ntu.edu.tw
}

\begin{abstract}
Stochastic Boolean satisfiability (SSAT) is an expressive language to formulate decision problems with randomness. Solving SSAT formulas has the same PSPACE-complete computational complexity as solving quantified Boolean formulas (QBFs). Despite its broad applications and profound theoretical values, SSAT has received relatively little attention compared to QBF. In this paper, we focus on exist-random quantified SSAT formulas, also known as E-MAJSAT, which is a special fragment of SSAT commonly applied in probabilistic conformant planning, posteriori hypothesis, and maximum expected utility. Based on clause selection, a recently proposed QBF technique, we propose an algorithm to solve E-MAJSAT. Moreover, our method can provide an approximate solution to E-MAJSAT with a lower bound when an exact answer is too expensive to compute. Experiments show that the proposed algorithm achieves significant performance gains and memory savings over the state-of-the-art SSAT solvers on a number of benchmark formulas, and provides useful lower bounds for cases where prior methods fail to compute exact answers.
\end{abstract}

\section{Introduction}

Stochastic Boolean satisfiability (SSAT) was first formulated in [Papadimitriou, 1985] and interpreted as games against nature. In an SSAT formula, a Boolean variable can be randomly quantified with probability $p$ by a randomized quantifier, which specifies that the variable evaluates to TRUE with probability $p$. With randomized quantifiers, SSAT serves as a natural formalism for an abundance of computational problems endowed with randomness, such as propositional probabilistic planning, trust management, and Bayesian network inference [Hnich et al., 2011; Littman et al., 2001; Majercik, 2009]. The verification problem of VLSI circuits with probabilistic errors has also been investigated under the framework of SSAT recently [Lee and Jiang, 2014]. From the perspective of computational complexity, SSAT lies in the

\footnotetext{
${ }^{*}$ The authors contributed equally to this work.
}

complexity class PSPACE-complete, the same as quantified Boolean formula (QBF). Therefore, advancing the scalability of SSAT solving not only benefits practical applications, but also has profound theoretical values.

In this paper, we focus on the exist-random quantified SSAT formulas of the form $\Phi=\exists X Y Y$. $\phi$, which is known as E-MAJSAT [Littman et al., 1998]. Computational problems, such as computing a maximum a posteriori (MAP) hypothesis or a maximum expected utility (MEU) solution [Dechter, 1998] in belief networks, and searching an optimal plan for probabilistic conformant planning domains [Littman et al., 1998], can be formulated with E-MAJSAT.

Among previous endeavors, several techniques have been investigated to solve SSAT. Prior work MAXPLAN [Majercik and Littman, 1998] utilizes Davis-Putnam-LogemannLoveland (DPLL) search [Davis et al., 1962] and improves its efficiency by considering pure variables, unit propagation, and subproblem memorization. DCSSAT [Majercik and Boots, 2005] enhances MAXPLAN by applying the divideand-conquer strategy to break SSAT formulas into many subproblems and solve them separately. The technique of knowledge compilation has also been exploited to solve EMAJSAT. ComP lan [Huang, 2006] compiles the problems into deterministic, decomposable negation normal form (dDNNF) [Darwiche, 2001; 2002], and performs a branchand-bound search. It is further improved in [Pipatsrisawat and Darwiche, 2009] by an enhanced bound computation method. Recent work [Fremont et al., 2017] proposed a maximum model counting algorithm for solving a special case of E-MAJSAT with all probabilities of randomized quantifiers equal to 0.5 .

Recently, modern SAT techniques have been utilized to solve random-exist quantified SSAT formulas in [Lee et al., 2017], which inspires us to examine the possibility of applying QBF solving techniques in the SSAT domain.

In this paper, we exploit the clause selection technique, which has been introduced in QBF solving recently [Janota and Marques-Silva, 2015; Rabe and Tentrup, 2015], and propose a clause containment learning method for E-MAJSAT solving. To the best of our knowledge, this paper is the first attempt to adopt approaches developed for QBF to solve SSAT. In addition to the learning technique, weighted model counting, which has been widely adopted in probabilistic inference [Chavira and Darwiche, 2008; Sang et al., 2005], is 
utilized in the proposed algorithm to compute probabilities.

We evaluate the proposed algorithm with a wide range of benchmarks including random formulas, planning problems, and probabilistic circuit verification. Experiments show that our method achieves significant performance gains and memory savings compared to the state-of-the-art SSAT solvers.

\section{Preliminaries}

We represent Boolean values TRUE and FALSE by symbols $T$ and $\perp$, respectively. In the sequel, a variable $x$ is assumed in the Boolean domain $\mathbb{B}=\{T, \perp\}$. A literal is a variable (called a positive literal) or the negation of a variable (called a negative literal). For a literal $l$, let $\operatorname{var}(l)$ denote the variable of $l$. Boolean connectives $\neg, \vee, \wedge, \Rightarrow, \equiv$ are interpreted in their conventional meanings. A clause is a disjunction of literals. A propositional Boolean formula $\phi$ is in Conjunctive Normal Form (CNF) if $\phi$ is a conjunction of clauses. A variable $x$ is said to be pure in a formula if its appearances in the formula are all in the positive phase $x$ or in the negative phase $\neg x$. A cube is a conjunction of literals. In the sequel, we assume propositional Boolean formulas are in CNF.

A Boolean formula $\phi$ over a set of variables $X=$ $\left\{x_{1}, \ldots, x_{n}\right\}$ defines a unique Boolean function $\mathbb{B}^{n} \rightarrow \mathbb{B}$. Let $\operatorname{vars}(\phi)$ denote the set of variables appearing in a Boolean formula $\phi$. An assignment $\tau$ over a set of variables $X \subseteq \operatorname{vars}(\phi)$ for a formula $\phi$ is a mapping $\tau: X \rightarrow \mathbb{B}$. An assignment $\tau: X \rightarrow \mathbb{B}$ is a complete assignment for formula $\phi$ if $X=\operatorname{vars}(\phi)$; otherwise, i.e., $X \subset \operatorname{vars}(\phi)$, it is a partial assignment. Given a Boolean formula $\phi$ and an assignment $\tau$ over vars $(\phi)$, the cofactor of $\phi$ under $\tau$, denoted by $\left.\phi\right|_{\tau}$, is derived by substituting every occurrence of each variable $x \in \operatorname{vars}(\phi)$ in $\phi$ by $\tau(x)$. If $\left.\phi\right|_{\tau}=\top$, we call $\tau$ a satisfying assignment of $\phi$. The satisfiability problem of a Boolean formula $\phi$ asks whether or not $\phi$ has a satisfying assignment. We write $\operatorname{SAT}(\phi)=\top$ to denote that $\phi$ is satisfiable. A satisfying assignment of $\phi$ is also called a model of $\phi$. On the other hand, if $\phi$ has no satisfying assignment, it is unsatisfiable and written as $\operatorname{SAT}(\phi)=\perp$. Given two Boolean formulas $\phi$ and $\psi$, we write $\phi \models \psi$ if every satisfying assignment for $\phi$ also satisfies $\psi$. In the sequel, we alternatively represent an assignment $\tau$ as a cube, a clause $C$ as a set of literals, and a CNF formula as a set of clauses.

\subsection{Stochastic Boolean Satisfiability}

A stochastic Boolean satisfiability (SSAT) formula is of the form

$$
\Phi=Q_{1} x_{1} \ldots Q_{n} x_{n} . \phi\left(x_{1}, \ldots, x_{n}\right),
$$

where $Q_{i} \in\left\{\exists, \mathrm{y}^{p_{i}}\right\}$ and $\phi$ is a quantifier-free Boolean formula. Symbol $\exists$ denotes the existential quantifier, and $\mathrm{y}^{p_{i}}$ denotes the randomized quantifier on $x_{i}$ for the probability of $x_{i}=\top$ equal to $p_{i} \in[0,1]$. Given an SSAT formula $\Phi$, the quantification structure $Q_{1} x_{1} \ldots Q_{n} x_{n}$ is called the pre$f i x$, and the Boolean formula $\phi$ is called the matrix.

Let $v$ be the outermost variable in the prefix of an SSAT formula $\Phi$. The satisfying probability of $\Phi$, denoted by $\operatorname{Pr}[\Phi]$, can be computed recursively by the following rules.

a) $\operatorname{Pr}[T]=1$, b) $\operatorname{Pr}[\perp]=0$,

c) $\operatorname{Pr}[\Phi]=\max \left\{\operatorname{Pr}\left[\left.\Phi\right|_{\neg v}\right], \operatorname{Pr}\left[\left.\Phi\right|_{v}\right]\right\}$, if $v$ is existentially quantified,

d) $\operatorname{Pr}[\Phi]=(1-p) \operatorname{Pr}\left[\left.\Phi\right|_{\neg v}\right]+p \operatorname{Pr}\left[\left.\Phi\right|_{v}\right]$, if $v$ is randomly quantified by $\mathrm{y}^{p}$.

In this paper, we focus on solving E-MAJSAT, the exist-random fragment of SSAT with the form $\Phi=$ $\exists X Y Y . \phi(X, Y)$, where $X$ and $Y$ are two disjoint sets of variables. The satisfying probability of $\Phi$ is obtained through maximizing $\operatorname{Pr}\left[\left.\Phi\right|_{\tau}\right]$, called the satisfying probability of $\Phi$ conditioned on $\tau$, over all assignments $\tau$ on $X$.

\subsection{Model Counting}

The model counting problem of a Boolean formula $\phi$ asks to find the number of satisfying assignments of $\phi$. Model counting algorithms can be classified into two categories: exact model counting and approximate model counting. The former computes the exact number of satisfying assignments of a formula; the latter computes an upper or a lower bound of the number of satisfying assignments with some confidence level. In its weighted version, a weight function $\omega$ is defined to map each variable $x \in \operatorname{vars}(\phi)$ to some weight $\omega(x) \in[0,1]$, which can be seen as the probability $\operatorname{Pr}[x=\top]$. The weight of a positive literal $x$ (resp. negative literal $\neg x$ ) of variable $x$ is defined to be $\omega(x)$ (resp. $1-\omega(x)$ ). The weight of an assignment is defined to be the product of the weights of its individual literals. The weight of a formula equals the summation of weights of its satisfying assignments.

Given an E-MAJSAT formula $\Phi=\exists X Y Y . \phi(X, Y)$ and an assignment $\tau$ on $X$, cofactoring the matrix with $\tau$ results in a formula $\left.\phi\right|_{\tau}$ referring only to variables in $Y$. The prefix $y Y$ induces a weight function $\omega: Y \rightarrow[0,1]$ for each variable $y \in Y$, where $\omega(y)$ equals the probability annotated on the randomized quantifier of $y$. As a result, the conditional satisfying probability $\operatorname{Pr}\left[\left.\Phi\right|_{\tau}\right]$, which equals the weight of the formula $\left.\phi\right|_{\tau}$ under the weight function $\omega$, can be obtained by invoking a weighted model counter on the formula $\left.\phi\right|_{\tau}$ with the weight function $\omega$. In the sequel, the invocation of a weighted model counter is expressed by WeightModelCount $\left(\mathrm{y} Y .\left.\phi\right|_{\tau}\right)$, which returns the conditional satisfying probability $\operatorname{Pr}\left[\mathrm{y} Y .\left.\phi\right|_{\tau}\right]$.

\subsection{Clause Selection}

Clause selection is a recently proposed technique for QBF solving [Janota and Marques-Silva, 2015; Rabe and Tentrup, 2015]. Given a CNF formula $\phi(X, Y)$ over a set of variables $X \cup Y$ with $X \cap Y=\emptyset$, we divide each clause $C \in \phi$ into two sub-clauses $C^{X}$ and $C^{Y}$, where $C^{X}$ (resp. $C^{Y}$ ) consists of the literals whose variables are in $X($ resp. $Y$ ). For example, for $C=\left(x_{1} \vee x_{2} \vee y_{1} \vee y_{2}\right)$, we have $C^{X}=\left(x_{1} \vee x_{2}\right)$ and $C^{Y}=\left(y_{1} \vee y_{2}\right)$. Clearly, $C=C^{X} \vee C^{Y}$.

A clause $C$ is said to be selected by an assignment $\tau$ over $X$ if every literal in $C^{X}$ is assigned to $\perp$ by $\tau ; C$ is said to be deselected by $\tau$ if some literal in $C^{X}$ is assigned to $\top$ by $\tau ; C$ is said to be undecided if it is neither selected nor deselected. We also use $\left.\phi\right|_{\tau}$ to denote the set of clauses selected by the assignment $\tau$. A selection variable $s_{C}$ is introduced for each 
clause $C$ and defined by $s_{C} \equiv \neg C^{X}$. Hence, $s_{C}$ is an indicator of the selection of clause $C$. That is, $s_{C}=\top$ (resp. $s_{C}=\perp$ ) indicates $C$ is selected (resp. deselected). Let $S$ be the set of selection variables for clauses in $\phi(X, Y)$. The formula $\psi(X, S)=\bigwedge_{C \in \phi}\left(s_{C} \equiv \neg C^{X}\right)$ is called the selection relation of $\phi(X, Y)$.

Example 1 Consider a CNF formula $\phi(X, Y)$ over two sets of variables $X=\left\{e_{1}, e_{2}, e_{3}\right\}$ and $Y=\left\{r_{1}, r_{2}, r_{3}\right\} . \phi(X, Y)$ consists of four clauses:

$$
\begin{aligned}
& C_{1}:\left(e_{1} \vee r_{1} \vee r_{2}\right) \\
& C_{2}:\left(e_{1} \vee e_{2} \vee r_{1} \vee r_{2} \vee \neg r_{3}\right) \\
& C_{3}:\left(\neg e_{2} \vee \neg e_{3} \vee r_{2} \vee \neg r_{3}\right) \\
& C_{4}:\left(\neg e_{1} \vee e_{3} \vee r_{3}\right)
\end{aligned}
$$

A selection variable $s_{i}$ is introduced for each clause, and $S=$ $\left\{s_{1}, s_{2}, s_{3}, s_{4}\right\}$. The selection relation $\psi(X, S)$ of $\phi(X, Y)$ equals

$$
\begin{aligned}
\psi(X, S)=\left(s_{1} \equiv \neg e_{1}\right) \wedge\left(s_{2} \equiv \neg e_{1} \wedge \neg e_{2}\right) & \wedge\left(s_{3} \equiv e_{2} \wedge e_{3}\right) \\
& \wedge\left(s_{4} \equiv e_{1} \wedge \neg e_{3}\right) .
\end{aligned}
$$

Consider the complete assignment $\tau_{1}=\neg e_{1} \neg e_{2} \neg e_{3}$ over $X$. It selects $C_{1}$ and $C_{2}$, and deselects $C_{3}$ and $C_{4}$, as can be seen from the selection relation cofactored by $\tau_{1}$, which results in $\left.\psi(X, S)\right|_{\tau_{1}}=s_{1} s_{2} \neg s_{3} \neg s_{4}$. Consider the partial assignment $\tau_{2}=\neg e_{1} e_{3}$ over $X$. It selects $C_{1}$, deselects $C_{4}$, and leaves $C_{2}$ and $C_{3}$ undecided. Notice that the two complete assignments $\neg e_{1} \neg e_{2} e_{3}$ and $\neg e_{1} e_{2} e_{3}$ consistent with $\tau_{2}$ select $\left\{C_{1}, C_{2}\right\}$ and $\left\{C_{1}, C_{3}\right\}$, respectively. The clause $C_{1}$ selected by the partial assignment $\tau_{2}$ lies in the intersection of the sets of clauses selected by the two complete assignments consistent with $\tau_{2}$.

\section{Clause Containment Learning}

Consider an E-MAJSAT formula $\Phi=\exists X \forall Y . \phi$. To obtain the satisfying probability of $\Phi$, it suffices to enumerate every assignment $\tau$ on $X$, and calculate the corresponding conditional satisfying probability $\operatorname{Pr}\left[\left.\Phi\right|_{\tau}\right]$. Clearly, the above brute-force approach is computationally expensive. Inspired by the idea of clause selection discussed above, we propose clause containment learning to prune the search space. The proposed learning technique deduces useful information after each trial of an assignment $\tau$ on $X$. The learnt information is recorded as blocking clauses to avoid wasteful exploration and thus accelerates the search process. The proposed learning technique is based on the following key observation.

Property 1 Given an E-MAJSAT formula $\Phi=$ $\exists X \mathrm{y} Y . \phi(X, Y)$ and two assignments $\tau_{1}$ and $\tau_{2}$ over $X$, we have

$$
\left(\left.\left.\phi\right|_{\tau_{2}} \models \phi\right|_{\tau_{1}}\right) \quad \Rightarrow \quad \operatorname{Pr}\left[\left.\Phi\right|_{\tau_{2}}\right] \leq \operatorname{Pr}\left[\left.\Phi\right|_{\tau_{1}}\right] .
$$

By Property 1 and clause selection, we propose clause containment learning as detailed below. After cofactoring $\phi$ with an arbitrary assignment $\tau_{1}$ over $X$, a set of clauses $\left.\phi\right|_{\tau_{1}}$ is selected. For any other assignment $\tau_{2}$ selecting every clause in $\left.\phi\right|_{\tau_{1}}$, i.e., $\left.\left.\phi\right|_{\tau_{1}} \subseteq \phi\right|_{\tau_{2}}$, we have $\left.\phi\right|_{\tau_{2}}=\left.\phi\right|_{\tau_{1}}$. Therefore, $\operatorname{Pr}\left[\left.\Phi\right|_{\tau_{2}}\right] \leq \operatorname{Pr}\left[\left.\Phi\right|_{\tau_{1}}\right]$ holds by Property 1 . Since the satisfying probability $\operatorname{Pr}\left[\left.\Phi\right|_{\tau_{2}}\right]$ is not greater than $\operatorname{Pr}\left[\left.\Phi\right|_{\tau_{1}}\right]$, the assignment $\tau_{2}$ is not worth trying. For all such assignments, they should be blocked after $\tau_{1}$ has been explored.

The core idea in the proposed clause containment learning is to block every unexplored assignment $\tau_{2}$ that selects a set of clauses $\left.\phi\right|_{\tau_{2}}$ containing a set of clauses $\left.\phi\right|_{\tau_{1}}$ previously selected by an explored assignment $\tau_{1}$. To block the assignment $\tau_{2}$, we enforce at least one of the clauses in $\left.\phi\right|_{\tau_{1}}$ to be deselected. Recall that the selection variable $s_{C}$ of clause $C$ valuates to $\perp$ if and only if $C$ is deselected. Therefore, a learnt clause, which is the disjunction of the negation of selection variables of clauses in $\left.\phi\right|_{\tau_{1}}$, is deduced to record this information. The above idea gives rise to the proposed algorithm in Figure 1 to solve E-MAJSAT formulas. (Lines 03, 07,08 and 11 describe enhancement techniques of the proposed algorithm, which will be discussed in Section 4.)

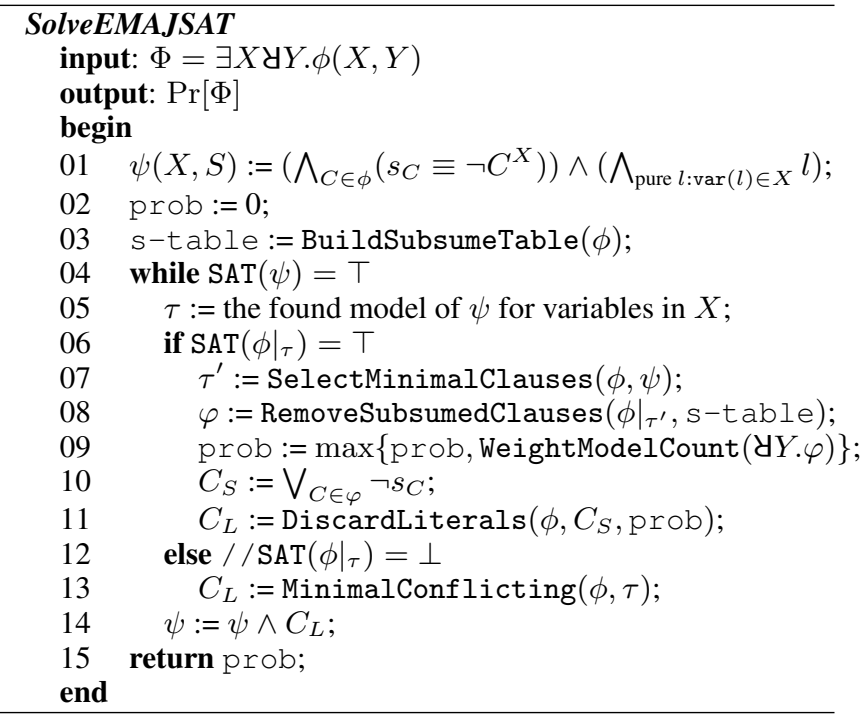

Figure 1: E-MAJSAT solving with clause containment learning.

The algorithm involves two SAT solvers: one works on the matrix $\phi(X, Y)$ of the input formula, and the other works on the selection relation $\psi(X, S)$ for clauses in $\phi(X, Y)$. Using the definition of selection variables, line 01 initializes the selection relation together with asserting the literals of pure $X$ variables. If a variable $e$ in $X$ is pure in $\phi$, assigning the literals of $e$ to $\top$ deselects the clauses containing $e$, and does not affect other clauses. Because the conditional satisfying probability is greater if less clauses are selected, we can always assert pure $X$ variables.

The selection relation is used to select different assignments $\tau$ over $X$. If $\left.\phi\right|_{\tau}$ is satisfiable, a weighted model counter is invoked to compute the conditional satisfying probability $\operatorname{Pr}\left[\mathrm{y} Y .\left.\phi\right|_{\tau}\right]$ under the assignment $\tau$. The blocking clause $C_{L}$ derived based on the proposed containment learning technique is conjoined with $\psi$ to prevent clauses in $\left.\phi\right|_{\tau}$ being simultaneously selected again.

On the other hand, suppose $\left.\phi\right|_{\tau}$ is unsatisfiable. We can deduce a conjunction of literals from $\tau$ responsible for the conflict by using a SAT solver to analyze the conflict (e.g., using the subroutine analyzeFinal in Minisat [Eén and 
Sörensson, 2003a; 2003b]). In general, the conjunction of literals may not be minimal, meaning that some literals can be discarded and the conflict remains unaffected. The subroutine MinimalConflicting makes the conjunction of literals responsible for the conflict minimal as follows. For each literal $l$ in the conjunction, temporarily drop $l$ and check whether $\phi(X, Y)$ is still unsatisfiable. If it is unsatisfiable, discard $l$; otherwise, keep $l$ in the conjunction. Repeating the above process for every literal makes the conjunction minimal. Complementing the minimal conjunction of literals yields a learnt clause, which is then conjoined with the selection relation to block assignments that make $\phi$ unsatisfiable.

When the selection relation becomes unsatisfiable, it indicates that the space spanned by variables $X$ has been completely searched. The algorithm will return the encountered maximum conditional satisfying probability, which equals the satisfying probability of the input E-MAJSAT formula.

We illustrate the working of algorithm SolveEMAJSAT in the following example.

Example 2 Continuing Example 1, we show how algorithm SolveEMAJSAT in Figure 1 (without the enhancement techniques) solves the E-MAJSAT instance $\Phi=$ $\exists e_{1} e_{2} e_{3} \mathrm{y}^{0.5} r_{1} r_{2} r_{3} . \phi(X, Y)$. Let the first tried assignment $\tau_{1}$ be $\neg e_{1} \neg e_{2} \neg e_{3}$, which selects $C_{1}, C_{2}$. The algorithm derives $\operatorname{Pr}\left[\mathrm{d}^{0.5} Y .\left.\phi\right|_{\tau_{1}}\right]=0.75$ by invoking the weighted model counter in line 09. The learnt clause $C_{L}=\left(\neg s_{1} \vee \neg s_{2}\right)$ is conjoined with $\psi$ to prevent $C_{1}$ and $C_{2}$ being selected simultaneously again. Suppose the second tried assignment $\tau_{2}$ is $\neg e_{1} e_{2} \neg e_{3}$, which selects $C_{1}$. The weighted model counter gives $\operatorname{Pr}\left[\mathrm{y}^{0.5} Y .\left.\phi\right|_{\tau_{2}}\right]=0.75$, and the learnt clause $C_{L}=$ $\left(\neg s_{1}\right)$ is conjoined with $\psi$ to prevent $C_{1}$ being selected again. Let the third tried assignment $\tau_{3}$ be $e_{1} e_{2} \neg e_{3}$, which selects $C_{4}$. The weighted model counter gives $\operatorname{Pr}\left[\mathrm{U}^{0.5} Y .\left.\phi\right|_{\tau_{3}}\right]=0.5$, and the learnt clause $C_{L}=\left(\neg s_{4}\right)$ is conjoined with $\psi$ to prevent $C_{4}$ being selected again. Let the fourth tried assignment $\tau_{4}$ be $e_{1} e_{2} e_{3}$, which selects $C_{3}$. The conditional satisfying probability $\operatorname{Pr}\left[\mathrm{y}^{0.5} Y .\left.\phi\right|_{\tau_{4}}\right]$ equals 0.75 , and the learnt clause $C_{L}=\left(\neg s_{3}\right)$ is conjoined with $\psi$ to prevent $C_{3}$ being selected again. Suppose the fifth tried assignment $\tau_{5}$ is $e_{1} \neg e_{2} e_{3}$, which deselects every clause, making $\left.\phi\right|_{\tau_{5}}=\top$ and $\operatorname{Pr}\left[\mathrm{d}^{0.5} Y .\left.\phi\right|_{\tau_{5}}\right]=1$. Since there is no selected clause, the learnt clause $C_{L}$ is empty, and the selection relation becomes unsatisfiable after being conjoined with an empty clause. The unsatisfiability of the selection relation reveals that the space spanned by variables $X$ has been exhaustively searched, and the algorithm returns the satisfying probability, which equals 1, of the E-MAJSAT instance.

\section{Enhancement Techniques}

The computational efficiency of algorithm SolveEMAJSAT is greatly affected by the strength of learnt clauses. We introduce three enhancement methods, minimal clause selection, clause subsumption, and partial assignment pruning, to strengthen the learnt clauses deduced by the proposed learning technique. In Figure 1, the enhancement techniques are executed by subroutines SelectMinimalClauses (in line 07), RemoveSubsumedClauses (in line 08), and
DiscardLiterals (in line 11), to be detailed in the following three sections, respectively.

\subsection{Minimal Clause Selection}

As discussed before, the selection relation $\psi(X, S)$ is in charge of choosing an assignment $\tau$ over variables $X$ and thus selects a set of clauses from the matrix $\phi(X, Y)$. However, the set of selected clauses may not be minimal, meaning that it is possible for another assignment $\tau^{\prime}$ to select a set of clauses contained in that selected by $\tau$, i.e., $\left.\left.\phi\right|_{\tau^{\prime}} \subset \phi\right|_{\tau}$. Notice that the length of a learnt clause equals the number of selected clauses. Therefore, selecting fewer clauses gives a stronger learnt clause, as well as a higher conditional satisfying probability. Starting from a set of initially selected clauses $\left.\phi\right|_{\tau}$, the first enhancement technique minimal clause selection decreases the number of selected clauses by making the set of initially selected clauses minimal as follows. The learnt clause is conjoined with $\psi(X, S)$ so that $\psi(X, S)$ is solved under the unit assumption [Eén and Sörensson, 2003b] to ensure that at least one of the currently selected clauses must be deselected in the future. The above process repeats until the selection relation becomes unsatisfiable.

\subsection{Clause Subsumption}

The second enhancement technique, clause subsumption, decreases the length of the learnt clause via examining the subsumption relation among the selected clauses. Recall that clause $C_{1}$ subsumes clause $C_{2}$ if every literal appears in $C_{1}$ also appears in $C_{2}$. Using the lookup table of the subsumption relation computed by subroutine BuildSubsumeTable, the procedure RemoveSubsumedClauses simplifies the set of selected clauses $\left.\phi\right|_{\tau}$ by removing subsumed clauses.

\subsection{Partial Assignment Pruning}

To illustrate the third enhancement technique partial assignment pruning, we first take a closer look at the learnt clause deduced by the proposed clause containment learning. Given a matrix $\phi(X, Y)$ and an assignment $\tau$ over $X$, a learnt clause consists of the negation of the selection variables of the selected clauses. For each selected clause $C$, if the selection variable $s_{C}$ is substituted by its definition $s_{C} \equiv \neg C^{X}$, the learnt clause $C_{L}$ becomes the disjunction of the sub-clauses $C^{X}$, i.e., $C_{L}=\bigvee_{\left.C \in \phi\right|_{\tau}} C^{X}$.

From the above illustration, we observe that the learnt clause can be strengthened as follows. First, temporarily discard some literal $l$ in the learnt clause. Second, invoke a weighted model counter to compute the conditional satisfying probability contributed by the selected clauses. Third, compare the probability to the current maximum satisfying probability. If the probability is no greater, literal $l$ is discarded; otherwise, it is kept in the clause. Fourth, repeat the above steps for other literals.

Benefiting from the three enhancement techniques, the efficiency of the proposed algorithm is greatly improved, as will be shown in our experiments.

We emphasize two more strengths of the proposed algorithm. First, during the computation, the proposed algorithm keeps deriving lower bounds for the satisfying probability, and the bounds gradually converge to the final answer. 
Therefore, in contrast to previous DPLL-based methods like DC-SSAT [Majercik and Boots, 2005], the proposed algorithm can be easily modified to solve approximate SSAT by returning the greatest encountered lower bound upon timeout. Second, the proposed algorithm is efficient in memory usage, since it stores the learnt information compactly via selection variables, and the weighted model counting is invoked on selected clauses, whose sizes are typically much smaller than that of original matrix.

\section{Experimental Results}

A prototyping program $^{1}$ of the proposed algorithm SolveEMAJSAT was implemented in the ABC [Mishchenko, 2005] environment with SAT solver Minisat-2.2 [Eén and Sörensson, 2003a]. For weighted model counting, we tried Cachet [Sang et al., 2004; 2005], but the overall performance was not satisfactory. Instead, we adopt the well-developed BDD package CUDD [Somenzi, 1998], and weighted model counting of the formula is done by traversing the BDD built from the formula, as proposed in [Darwiche and Marquis, 2002]. The experiments were conducted on a Linux machine with an Intel Xeon 2.1 GHz CPU and 126 GB RAM.

We evaluated the proposed algorithm on both random $k$ $\mathrm{CNF}$ and application formulas. We compared the proposed approach to the state-of-the-art SSAT solver DC-SSAT, the maximum model counter MAXCOUNT [Fremont et al., 2017], which relies on the approximate counter APPROXMC [Chakraborty et al., 2016], and the E-MAJSAT solver ComPlan. As ComPlan is not publicly available, we used the CNF-to-d-DNNF compilation time of $\mathrm{c} 2 \mathrm{~d}$ [Darwiche, 2002], a key step in ComPlan, to estimate the performance of Complan. Because MAXCOUNT can only handle randomized quantifiers with probability 0.5 , to compare MAXCOUNT against other methods we convert formulas involving randomized quantifiers with probabilities not equal to 0.5 into equivalent ones with all probabilities equal to 0.5 by the conversion method proposed in [Lee and Jiang, 2014].

In the sequel, our proposed approach with all three enhancement techniques enabled, DC-SSAT, MAXCOUNT, and the CNF-to-d-DNNF compiler are referred to as erSSAT, Dc, Max, and c2d, respectively. A runtime limit of 1000 seconds was imposed on each formula, and the symbol "-" denotes timeout. We did not impose a memory limit, but recorded the maximum memory usage during execution.

\subsection{Random $k$-CNF Formulas}

The random $k$-CNF formulas were generated by the $\mathrm{cn} f \mathrm{gen}$ package [Lauria, 2012]. A collection of 280 formulas were generated with $k$, i.e., the number of literals in a clause, ranging in $\{3,4,5,6,7,8,9\}$, the number of variables ranging in $\{10,20,30,40,50\}$, and clause-to-variable ratio ranging in $\{k-1, k, k+1, k+2\}$. Two formulas were generated for each parameter combination. To convert the propositional formulas into E-MAJSAT formulas, half of the variables are existentially quantified, and the rest are randomly quantified with probability 0.5 .

\footnotetext{
${ }^{1}$ Available at https://github.com/nianzelee/ssatABC.git
}

Among the collection of 280 formulas, erSSAT solved 216 instances exactly and computed lower bounds for the rest 64 instances with confidence level 1, while Dc and c $2 \mathrm{~d}$ solved 210 and 209 instances, respectively. On the other hand, Max computed lower bounds for 257 instances with confidence level greater than 0.8. Moreover, the maximum memory usage of erSSAT is two orders (resp. one order) of magnitude less than that of $D_{C}$ (resp. $c 2 d$ ). As the runtime and memory usage of ComPlan are bounded below by $\mathrm{c} 2 \mathrm{~d}$, we conclude that erSSAT has the best performance among compared solvers over random formulas.

\subsection{Application Formulas}

We collected seven families of application formulas for evaluation. The first two families, Toilet-A and Conformant, are exist-forall-exist quantified QBFs from [Giunchiglia et al., 2005]. We converted them into exist-random-exist SSAT formulas by changing universal quantifiers to randomized ones with probabilities 0.5. The third family Sand-Castle is a probabilistic conformant planning problem, which was encoded as an E-MAJSAT formula in [Majercik and Littman, 1998]. The fourth, fifth, and sixth families are taken from [Fremont et al., 2017], which encode the maximum satisfiability (MaxSat), quantitative information flow (QIF), and program synthesis (PS) problems, respectively, into maximum model counting. The last family is taken from [Lee and Jiang, 2014], which encodes the maximum probabilistic equivalence checking (MPEC) of analyzing the maximum probability for a probabilistic circuit to produce erroneous outputs.

Table 1 shows the results, where benchmarks statistics, including the numbers of variables (\#V), clauses (\#C), outermost existentially quantified variables $\left(\# \mathrm{E}_{1}\right)$, randomized quantified variables (\#R), and innermost existentially quantified variables $\left(\# E_{2}\right)$, are reported. In the table, runtime information is reported in seconds. For erSSAT, the obtained lower bound (LB), the time $\left(\mathrm{T}_{1}\right)$ spent to first reach the obtained LB, and the entire runtime $\left(\mathrm{T}_{2}\right)$, are reported. For DC, the exact probability $(\mathrm{Pr})$ and runtime $(\mathrm{T})$ are reported. As DC is an exact SSAT solver, either it terminates and returns an answer, or it times out without producing any information. For Max, the lower bound (LB), confidence level (CL) of LB, and the rumtime $(\mathrm{T})$ are reported. For $\mathrm{C} 2 \mathrm{~d}$, the time $(\mathrm{T})$ spent on compiling a formula into d-DNNF is reported. A “-” entry in $\mathrm{T}, \mathrm{T}_{1}$, or $\mathrm{T}_{2}$ indicates that the time limit was reached. We note that, if $\mathrm{T}_{2}$ of erSSAT is not a "-", the corresponding LB is the exact answer to the formula. Also the LB values obtained by erSSAT are of confidence level 1 .

The results suggest that erSSAT was able to quickly derive tight lower bounds in families Toilet-A, Conformant, Sand-Castle, MaxSat, and MPEC. Specifically, for Toilet-A, erSSAT generally spent less time than Max did to derive the same lower bounds while Max timed out on four larger cases. For Conformant, erSSAT derived lower bounds at the order of $10^{-1}$ for all formulas, while Dc, Max, and $\mathrm{c} 2 \mathrm{~d}$ timed out on most of them. For Sand-Castle, erSSAT exactly solved three formulas, and derived lower bounds greater than 0.99 for the rest. In contrast, Dc and c $2 \mathrm{~d}$ outperformed erSSAT on these instances because they are specially designed for solving probabilistic conformant planning tasks. On the other 
Proceedings of the Twenty-Seventh International Joint Conference on Artificial Intelligence (IJCAI-18)

\begin{tabular}{|c|c|c|c|c|c|c|c|c|c|c|c|c|c|c|c|}
\hline \multicolumn{7}{|c|}{ benchmark statistics } & \multicolumn{3}{|c|}{ erSSAT } & \multicolumn{2}{|c|}{$\overline{D C}$} & \multicolumn{3}{|c|}{ Max } & c2d \\
\hline family & formula & $\# \mathrm{~V}$ & \#C & $\mathrm{HE}_{1}$ & \#R & $\# \mathrm{E}_{2}$ & LB & $\mathrm{T}_{1}$ & $\mathrm{~T}_{2}$ & $\operatorname{Pr}$ & $\mathrm{T}$ & LB & $\mathrm{CL}$ & $\mathrm{T}$ & $\mathrm{T}$ \\
\hline \multirow{7}{*}{ Toilet-A } & $10 \_01.3$ & 106 & 10604 & 33 & 10 & 63 & $1.95 \mathrm{e}-3$ & 0 & 27 & $1.95 \mathrm{e}-3$ & 13 & $1.95 \mathrm{e}-3$ & 1.00 & 36 & 3 \\
\hline & $10 \_01.5$ & 170 & 10902 & 55 & 10 & 105 & 3.91e-3 & 19 & 577 & $3.91 \mathrm{e}-3$ & 208 & $3.91 \mathrm{e}-3$ & 1.00 & 67 & 5 \\
\hline & 10_01.7 & 234 & 11200 & 77 & 10 & 147 & $7.81 \mathrm{e}-3$ & 179 & - & - & - & $7.81 \mathrm{e}-3$ & 1.00 & 294 & 19 \\
\hline & 10_05.2 & 170 & 11315 & 110 & 10 & 50 & $3.13 \mathrm{e}-2$ & 565 & - & - & - & - & - & - & \\
\hline & $10 \_05.3$ & 250 & 12000 & 165 & 10 & 75 & $1.56 \mathrm{e}-2$ & 0 & - & - & - & - & - & - & 244 \\
\hline & 10_05.4 & 330 & 12685 & 220 & 10 & 100 & $1.56 \mathrm{e}-2$ & 888 & - & - & - & - & - & - & - \\
\hline & $10 \_10.2$ & 290 & 12840 & 220 & 10 & 60 & 1.00 & 3 & 3 & - & - & - & - & - & 181 \\
\hline \multirow{7}{*}{ Conformant } & blocks_enc_2_b4 & 3043 & 57130 & 1248 & 7 & 1788 & $4.38 \mathrm{e}-1$ & 341 & - & - & - & - & - & - & - \\
\hline & cube_c7_ser-23 & 1479 & 15164 & 138 & 9 & 1332 & $3.38 \mathrm{e}-1$ & 620 & - & - & - & - & - & - & - \\
\hline & cube_c7_ser-opt-24 & 1542 & 15510 & 144 & 9 & 1389 & $3.44 \mathrm{e}-1$ & 679 & - & - & - & - & - & - & - \\
\hline & cube_c9_par-10 & 847 & 24106 & 60 & 10 & 777 & $2.90 \mathrm{e}-1$ & 185 & - & - & - & $2.92 \mathrm{e}-1$ & 1.00 & 802 & - \\
\hline & cube_c9_par-opt-11 & 928 & 24548 & 66 & 10 & 852 & $2.89 \mathrm{e}-1$ & 192 & - & - & - & - & - & - & - \\
\hline & emptyroom_e3_ser-20 & 982 & 6286 & 80 & 6 & 896 & $1.88 \mathrm{e}-1$ & 869 & - & - & - & - & - & - & - \\
\hline & ring_r4_ser-opt-11 & 373 & 5333 & 44 & 9 & 320 & $4.96 \mathrm{e}-1$ & 506 & - & - & - & $4.53 \mathrm{e}-1$ & 1.00 & 102 & 29 \\
\hline \multirow{7}{*}{ Sand-Castle } & SC-11 & 101 & 201 & 22 & 55 & 24 & $9.77 \mathrm{e}-1$ & 32 & 50 & $9.77 \mathrm{e}-1$ & 0 & - & - & - & 0 \\
\hline & SC-12 & 110 & 219 & 24 & 60 & 26 & $9.84 \mathrm{e}-1$ & 133 & 187 & $9.84 \mathrm{e}-1$ & 0 & - & - & - & 0 \\
\hline & SC-13 & 119 & 237 & 26 & 65 & 28 & $9.89 \mathrm{e}-1$ & 441 & 619 & $9.89 \mathrm{e}-1$ & 0 & - & - & - & 0 \\
\hline & SC-14 & 128 & 255 & 28 & 70 & 30 & $9.92 \mathrm{e}-1$ & 632 & - & $9.92 \mathrm{e}-1$ & 1 & - & - & - & 0 \\
\hline & SC-15 & 137 & 273 & 30 & 75 & 32 & $9.93 \mathrm{e}-1$ & 979 & - & $9.94 \mathrm{e}-1$ & 1 & - & - & - & 1 \\
\hline & SC-16 & 146 & 291 & 32 & 80 & 34 & $9.94 \mathrm{e}-1$ & 785 & - & $9.96 \mathrm{e}-1$ & 3 & - & - & - & 0 \\
\hline & SC-17 & 155 & 309 & 34 & 85 & 36 & $9.94 \mathrm{e}-1$ & 654 & - & $9.97 \mathrm{e}-1$ & 6 & - & - & - & 1 \\
\hline MaxSat & keller4.clq & 120 & 1212 & 43 & 15 & 62 & $9.76 \mathrm{e}-1$ & 0 & 0 & - & - & $9.13 \mathrm{e}-1$ & 0.82 & 5 & 1 \\
\hline \multirow{7}{*}{ QIF } & backdoor- $2 \times 16-8$ & 200 & 272 & 32 & 32 & 136 & $5.96 \mathrm{e}-8$ & 0 & - & - & - & $5.96 \mathrm{e}-8$ & 1.00 & 9 & 1 \\
\hline & backdoor-32-24 & 147 & 76 & 32 & 32 & 83 & 1.00 & 0 & 0 & - & - & $1.95 \mathrm{e}-3$ & 0.82 & 601 & 0 \\
\hline & bin-search-16 & 1448 & 5825 & 16 & 16 & 1416 & $1.95 \mathrm{e}-3$ & 106 & - & - & - & $9.85 \mathrm{e}-1$ & 0.91 & 230 & - \\
\hline & CVe-2007-2875 & 784 & 1740 & 32 & 32 & 720 & 1.00 & 2 & 2 & - & - & $9.85 \mathrm{e}-1$ & 0.82 & 13 & 342 \\
\hline & pwd-backdoor & 400 & 609 & 64 & 64 & 272 & 0.00 & - & - & - & - & $9.85 \mathrm{e}-1$ & 0.99 & 93 & 1 \\
\hline & reverse 2 & 333 & 293 & 32 & 32 & 269 & $2.98 \mathrm{e}-7$ & 271 & - & - & - & - & - & - & 2 \\
\hline & reverse & 229 & 293 & 32 & 32 & 165 & $5.96 \mathrm{e}-7$ & 839 & - & - & - & - & - & - & 2 \\
\hline \multirow{7}{*}{ PS } & ConcreteActService & 4836 & 17866 & 71 & 37 & 4728 & 0.00 & - & - & - & - & $9.60 \mathrm{e}-1$ & 0.82 & 52 & - \\
\hline & IssueServiceImpl & 3625 & 13028 & 77 & 29 & 3519 & 0.00 & - & - & - & - & $9.06 \mathrm{e}-1$ & 0.82 & 34 & - \\
\hline & IterationService & 4167 & 15264 & 70 & 34 & 4063 & 0.00 & - & - & - & - & $9.70 \mathrm{e}-1$ & 0.82 & 47 & - \\
\hline & LoginService & 5229 & 21566 & 92 & 27 & 5110 & 0.00 & - & - & - & - & $9.45 \mathrm{e}-1$ & 0.82 & 56 & - \\
\hline & PhaseService & 4167 & 15264 & 70 & 34 & 4063 & 0.00 & - & - & - & - & $9.70 \mathrm{e}-1$ & 0.82 & 47 & - \\
\hline & ProcessBean & 9880 & 41451 & 166 & 39 & 9675 & 0.00 & - & - & - & - & $9.27 \mathrm{e}-1$ & 0.82 & 126 & - \\
\hline & UserServiceImpl & 4019 & 14657 & 87 & 31 & 3901 & 0.00 & - & - & - & - & $9.22 \mathrm{e}-1$ & 0.82 & 43 & - \\
\hline \multirow{7}{*}{ MPEC } & $\mathrm{c} 499(2.34 \mathrm{e}-1)$ & 217 & 522 & 41 & 2 & 174 & $2.34 \mathrm{e}-1$ & 0 & 0 & $2.34 \mathrm{e}-1$ & 0 & $2.34 \mathrm{e}-1$ & 1.00 & 0 & 2 \\
\hline & $\mathrm{c} 880(2.34 \mathrm{e}-1)$ & 451 & 1167 & 60 & 2 & 389 & $1.25 \mathrm{e}-1$ & 0 & - & - & - & $1.25 \mathrm{e}-1$ & 1.00 & 14 & 72 \\
\hline & $\mathrm{c} 1355(3.30 \mathrm{e}-1)$ & 771 & 2181 & 41 & 3 & 727 & $3.30 \mathrm{e}-1$ & 0 & - & - & - & $3.30 \mathrm{e}-1$ & 1.00 & 41 & 10 \\
\hline & $\mathrm{c} 1908(2.34 \mathrm{e}-1)$ & 270 & $\begin{array}{r}2101 \\
705\end{array}$ & 33 & 2 & 235 & $2.34 \mathrm{e}-1$ & 23 & - & $2.34 \mathrm{e}-1$ & 91 & $1.25 \mathrm{e}-1$ & 1.00 & 1 & 3 \\
\hline & $\mathrm{c} 3540(1.25 \mathrm{e}-1)$ & 321 & 807 & 50 & 2 & 269 & $1.25 \mathrm{e}-1$ & 0 & - & $1.25 \mathrm{e}-1$ & 92 & $1.25 \mathrm{e}-1$ & 1.00 & 2 & 3 \\
\hline & c5315(7.37e-1) & 918 & 2190 & 178 & 10 & 730 & $4.14 \mathrm{e}-1$ & 154 & - & - & - & $6.27 \mathrm{e}-1$ & 0.82 & 63 & 217 \\
\hline & c7552(4.87e-1) & 648 & 1308 & 207 & 5 & 436 & $2.34 \mathrm{e}-1$ & 0 & - & - & - & $2.18 \mathrm{e}-1$ & 0.82 & 66 & 5 \\
\hline \multicolumn{7}{|c|}{ Maximum memory usage (GB) } & & & 2.2 & & 38.6 & & & 0.2 & 4.2 \\
\hline
\end{tabular}

Table 1: Results of Application Formulas

hand, Max failed in deriving lower bounds for all the formulas. For families QIF and PS, erSSAT did not perform as good as Max due to the fact that the formulas in these two families share the property that only very few assignments to the outermost existentially quantified variables can lead to large satisfying probabilities close to 1 . It is not surprising as Max is particularly designed for such formulas.

In terms of memory usage, erSSAT is more efficient than Dc and $\mathrm{c} 2 \mathrm{~d}$ while Max used the least amount.

\subsection{Analysis of Enhancement Techniques}

To analyze the effect of the proposed enhancement techniques on computational efficiency, we tested our program under different configurations over random $k$-CNF formulas. Let the enabled enhancement techniques be indicated by letter $\mathrm{m}$ for minimal clause selection, $\mathrm{s}$ for clause subsumption, and $\mathrm{p}$ for partial assignment pruning. On average, erSSAT- $\{\mathrm{p}\}$ achieved $31 \%$ speedup over erSSAT; erSSAT $-\{\mathrm{m}, \mathrm{p}\}$ achieved $11 \%$ speedup over erSSAT $-\{\mathrm{p}\} ; \operatorname{erSSAT}-\{\mathrm{m}, \mathrm{s}, \mathrm{p}\}$ achieved $2 \%$ speedup over erSSAT $-\{\mathrm{m}, \mathrm{p}\}$.

For application formulas, the enhancement techniques in general do not produce consistent runtime improvements due to their computational overheads. However they helped in exact solving of more formulas within the timeout limit. The results suggest the benefit of the three enhancement techniques to the efficiency of the proposed algorithm.

\section{Conclusions}

We developed a new approach to solving E-MAJSAT formulas. In contrast to prior methods based on DPLL search or knowledge compilation, we proposed the clause containment learning technique, inspired by clause selection recently developed in QBF evaluation, and design a novel algorithm to solve E-MAJSAT efficiently. Under the framework of clause containment learning, three enhancement techniques were proposed to improve the computational efficiency. Experiment results show the benefit of our method. For future work, we intend to solve SSAT with general prefix structure.

\section{Acknowledgements}

This work was supported in part by the Ministry of Science and Technology of Taiwan under grants 104-2628E-002-013-MY3, 105-2221-E-002-196-MY3, 105-2923-E002-016-MY3, and 106-2912-E-002-002-MY3. 


\section{References}

[Chakraborty et al., 2016] S. Chakraborty, K. Meel, and M. Vardi. Algorithmic improvements in approximate counting for probabilistic inference: From linear to logarithmic SAT calls. In Proc. IJCAI, pages 3569-3576, 2016.

[Chavira and Darwiche, 2008] M. Chavira and A. Darwiche. On probabilistic inference by weighted model counting. Artificial Intelligence, 172(6-7):772-799, 2008.

[Darwiche and Marquis, 2002] A. Darwiche and P. Marquis. A knowledge compilation map. Journal of Artificial Intelligence Research, 17:229-264, 2002.

[Darwiche, 2001] A. Darwiche. Decomposable negation normal form. Journal of the ACM, 48(4):608-647, 2001.

[Darwiche, 2002] A. Darwiche. A compiler for deterministic, decomposable negation normal form. In Proc. AAAI, pages 627-634, 2002.

[Davis et al., 1962] M. Davis, G. Logemann, and D. Loveland. A machine program for theorem-proving. Соттиnications of the ACM, 5(7):394-397, 1962.

[Dechter, 1998] R. Dechter. Bucket elimination: A unifying framework for probabilistic inference. In Learning in Graphical Models, pages 75-104. Springer, 1998.

[Eén and Sörensson, 2003a] N. Eén and N. Sörensson. An extensible SAT-solver. In Proc. SAT, pages 502-518, 2003.

[Eén and Sörensson, 2003b] N. Eén and N. Sörensson. Temporal induction by incremental SAT solving. Electronic Notes in Theoretical Computer Science, 89(4):543-560, 2003.

[Fremont et al., 2017] D. Fremont, M. Rabe, and S. Seshia. Maximum Model Counting. In AAAI, pages 3885-3892, 2017.

[Giunchiglia et al., 2005] E. Giunchiglia, M. Narizzano, L. Pulina, and A. Tacchella. Quantified Boolean formulas satisfiability library (QBFLIB), 2005. https://www.qbflib.org.

[Hnich et al., 2011] B. Hnich, R. Rossi, A. Tarim, and S. Prestwich. A survey on CP-AI-OR hybrids for decision making under uncertainty. In Hybrid Optimization, pages 227-270. Springer, 2011.

[Huang, 2006] J. Huang. Combining knowledge compilation and search for conformant probabilistic planning. In Proc. ICAPS, pages 253-262, 2006.

[Janota and Marques-Silva, 2015] M. Janota and J. Marques-Silva. Solving QBF by clause selection. In Proc. IJCAI, pages 325-331, 2015.

[Lauria, 2012] M. Lauria. CNFgen: Combinatorial benchmarks for SAT solvers, 2012. https://massimolauria.github.io/cnfgen/.

[Lee and Jiang, 2014] N.-Z. Lee and J.-H. Jiang. Towards formal evaluation and verification of probabilistic design. In Proc. ICCAD, pages 340-347, 2014.
[Lee et al., 2017] N.-Z. Lee, Y.-S. Wang, and J.-H. Jiang. Solving stochastic Boolean satisfiability under randomexist quantification. In Proc. IJCAI, pages 688-694, 2017.

[Littman et al., 1998] M. Littman, J. Goldsmith, and M. Mundhenk. The computational complexity of probabilistic planning. Journal of Artificial Intelligence Research, 9(1):1-36, 1998.

[Littman et al., 2001] M. Littman, S. Majercik, and T. Pitassi. Stochastic Boolean satisfiability. Journal of Automated Reasoning, 27(3):251-296, 2001.

[Majercik and Boots, 2005] S. Majercik and B. Boots. DCSSAT: A divide-and-conquer approach to solving stochastic satisfiability problems efficiently. In Proc. AAAI, page 416, 2005.

[Majercik and Littman, 1998] S. Majercik and M. Littman. MAXPLAN: A new approach to probabilistic planning. In Proc. AIPS, pages 86-93, 1998.

[Majercik, 2009] S. Majercik. Stochastic Boolean satisfiability. Handbook of Satisfiability, 185:887-925, 2009.

[Mishchenko, 2005] A. Mishchenko. ABC: A system for sequential synthesis and verification, 2005. https://github.com/berkeley-abc/abc.

[Papadimitriou, 1985] C. Papadimitriou. Games against nature. Journal of Computer and System Sciences, 31(2):288-301, 1985.

[Pipatsrisawat and Darwiche, 2009] K. Pipatsrisawat and A. Darwiche. A new d-DNNF-based bound computation algorithm for functional E-MAJSAT. In Proc. IJCAI, pages 590-595, 2009.

[Rabe and Tentrup, 2015] M. Rabe and L. Tentrup. CAQE: A certifying QBF solver. In Proc. FMCAD, pages 136$143,2015$.

[Sang et al., 2004] T. Sang, F. Bacchus, P. Beame, H. Kautz, and T. Pitassi. Combining component caching and clause learning for effective model counting. In Proc. SAT, pages 20-28, 2004.

[Sang et al., 2005] T. Sang, P. Beame, and H. Kautz. Performing Bayesian inference by weighted model counting. In Proc. AAAI, pages 475-481, 2005.

[Somenzi, 1998] F. Somenzi. CUDD: CU decision diagram package release 2.3.0, 1998 . 